\title{
Assessing the effectiveness of ketorolac in pain management of traumatic injuries in prehospital emergency care services
}

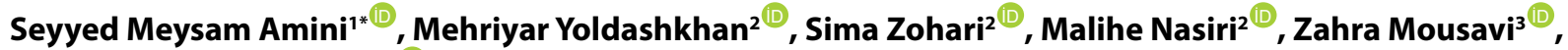 \\ Seyed Mohammad Amini ${ }^{4}$
}

'Student Research Committee, School of Nursing and Midwifery, Shahid Beheshti University of Medical Sciences, Tehran, Iran ${ }^{2}$ Department of Medical Surgical Nursing, School of Nursing and Midwifery, Shahid Beheshti University of Medical Sciences, Tehran, Iran

${ }^{3}$ Department of Nursing and Midwifery, Lorestan University of Medical Sciences, Khorramabad, Iran

${ }^{4}$ Radiation Biology Research Center, Iran University of Medical Sciences,Tehran, Iran

\begin{abstract}
Received: 15 January 2021
Accepted: 10 April 2021

Published online: 23 April 2021

*Corresponding author:

Seyyed Meysam Amini,

Student Research Committee,

School of Nursing and Midwifery,

Shahid Beheshti University of

Medical Sciences, Tehran, Iran

Email: sm.amini1987@gmail.com

Competing interests: None.

Funding information: None.

Citation: Amini SM, Yoldashkhan M Zohari S, Nasiri M, Mousavi Z, Amin $\mathrm{SM}$. Assessing the effectiveness of ketorolac in pain management of traumatic injuries in prehospital emergency care services. Journal of Emergency Practice and Trauma 2022; 8(1): 8-12. doi: 10.34172/ jept.2021.01
\end{abstract}

\begin{abstract}
Objective: Pain is usually one of the most common symptoms among all traumatic injuries. One of the drugs that has recently entered the Iranian prehospital emergency system is ketorolac. Ketorolac is a non-steroidal anti-inflammatory drug (NSAID) that inhibits prostaglandin synthesis by inhibiting cyclooxygenase activity and reduces pain and inflammation. It can be considered as the strongest analgesic drug in this category. The aim of this study was to evaluate the effectiveness of ketorolac in pain management of traumatic injuries in a prehospital setting.

Methods: This descriptive cross-sectional study was undertaken to evaluate the effectiveness of a treatment method in the pain management of traumatic injuries in the prehospital environment of Lorestan province in Iran. We included 134 injured patients from road emergency bases of Lorestan province. In order to control the pain of these injured patients, $30 \mathrm{mg}$ of ketorolac was injected slowly intravenously in one minute. Pain was measured in the time interval of zero minutes (before injection), 15 minutes, 30 minutes, and 45 minutes by visual acuity scale (VAS). The analysis of the obtained data was performed by SPSS software version 23.

Results: The mean age of participants was $37.42 \pm 23.6$. There were 47 female injuries (35.1\%) and 87 male injuries (64.9\%). In terms of pain intensity, 49 patients $(36.6 \%)$ had moderate pain $(\mathrm{VAS}=4-6)$ and 85 patients $(63.4 \%)$ had severe pain (VAS $=7-10)$. The average of pain relief in the injured group with severe and moderate pain 15 minutes after the injection was 0.471 and $0.878(P=0.001)$, respectively. In addition, the mean of pain relief in the injured group with severe and moderate pain 30 minutes after the injection was 1.124 and 1.796 , respectively $(P=0.001)$. Pain reduction in the group of severely injured patients with moderate pain in the first 30 minutes was statistically significant.

Conclusion: Findings revealed that ketorolac is a suitable drug in pain management for trauma patients with moderate and severe pain in trauma patients. On the other hand, due to the peak effect (more than 30 minutes), it is not an effective drug for trauma patients with severe pain in a short period transferred to the prehospital environment.

Keywords: Ketorolac, Traumatic injuries, Pain management, Pre-hospital emergency
\end{abstract}

\section{Introduction}

Trauma injuries have been on the rise in recent years, not only in developed countries but around the world (1). Pain control and the management in trauma patients in the early stages in prehospital emergency services and emergency department (ED) is inversely related to the duration of hospitalization of the trauma patients (2). Pain management can be effective in saving patients' lives as it can diminish severe pain which can have an effect on the blood pressure, respiration rate, and heart rate. Severe pain causes multiple physiological and pathological responses in the patient that stems from insufficient pain control and can lead to organ failure and in some cases even death (3). To choose the right analgesic for pain management, various parameters must be considered, the most important of which include: the incidence of side effects, rapid onset of action and drug interaction with other medications. (4). 
One of the most common methods of pain management for patients is the use of strong opioid analgesics, but the use of this group of drugs can cause common side effects even in short-term use, such as nausea and dizziness and respiratory rhythm disorders. This is followed by respiratory arrest due to suppression of the respiratory system, or long-term use such as drug dependency on the patient (5). One of the drugs that has recently entered the Iranian prehospital emergency system is ketorolac. ketorolac is a non-steroidal anti-inflammatory drug (NSAID) (6). Ketorolac has several uses. Being an analgesic is one of the most common uses of ketorolac. It can be considered as the strongest analgesic in this class of drugs (7). The present study was conducted to evaluate the effectiveness of ketorolac injections in controlling the pain of traumatic patients in a prehospital setting.

\section{Methods}

This descriptive study was conducted to evaluate the efficacy of ketorolac in the pain management of trauma patients in the Road Emergency Bases (REB) of Lorestan Emergency Medical Center between 2018 and 2019. We used convenience sampling according to the inclusion and exclusion criteria of the study. The study protocol was approved by the ethics committee of Shahid Beheshti University of Medical Sciences, Iran with code number IR.SBMU.PHARMACY. REC.1397.265.

We included trauma patients in the age range of 15-65 years who suffered from penetrating or non-penetrating trauma in the four extremities and needed emergency medical services (EMS) in Lorestan province. The inclusion criteria encompassed having pain with the intensity of at least 4 or more according to the visual analogue scale (VAS) and normal level of consciousness (Glasgow Coma Scale of 15). We excluded patients with a history of liver and kidney failure, patients with coagulation hemorrhagic disease, patients with recent history of surgery, pregnant women, patients with head trauma, history of any drug allergies, history of addiction, any sensory disturbances or spinal cord injuries caused by trauma, extensive trauma with active bleeding, patients with severe and decreased level of consciousness. Demographic information and vital signs as well as the intensity of pain were recorded by an emergency medicine technician. In addition, the emergency medicine technician injected $30 \mathrm{mg}$ of ketorolac in order to manage the pain. Ketorolac was intravenously injected slowly $(30 \mathrm{mg} / \mathrm{min})$. During the transfer of the injured patients to the nearest trauma center, the vital signs and the amount of pain of the injured patient was recorded by VAS every 15 minutes (till 45 minutes) after the injection of the analgesic drug.

Statistical analysis was performed using SPSS software version 23. Chi-square and $t$ test were used to compare qualitative and quantitative variables, respectively. In order to compare pain relief in both groups, we applied $t$ test analysis.
The minimum acceptable amount for pain relief according to the VAS scale is $13 \mathrm{~mm}$. The degree of pain relief up to $30 \mathrm{~mm}$ is medically significant. According to the VAS scale, the severity of pain was categorized into three groups based on severity: mild, moderate, and severe. (8). According to VAS, pain intensity of 0-39 was considered as mild, 40-69 as moderate and 70-100 as severe (9).

\section{Results}

Finally, 134 trauma patients with the mean age of $37.42 \pm 23.6$ (15-65) years were included in the study. The maximum age range was 35-45 years (\%31/3). Eightyseven males (64.9\%) and 47 females (35.1\%). Before the injection of ketorolac, the average degree of the pain was 7.667. Fifteen minutes after the injection, the average degree of pain was 6.567 . The value of the pain decreased to 5.573 at 30 minutes. In the final assessment, 45 minutes after the injection, the value of the pain decreased to 4 . 444.The average of pain in both males and females was 7.17 and 7.17 , respectively, with no significant statistical differences $(P=0.796)$. Ninety-nine patients suffered from transportation-related accidents $(73.9 \%)$ and 35 patients suffered from non-transportation-related accidents (26.1\%). There were no significant statistical differences between the two groups concerning the pain intensity $(P=0.93)$.

In terms of pain intensity, 49 patients $(36.6 \%)$ had moderate pain (VAS $=4-6)$ with the mean pain score of 5.122 , and 85 patients $(63.4 \%)$ had severe pain (VAS $=7-10$ ) with the mean pain score of 8.376 . The heart rate of trauma patients with severe and moderate pain was 90.1272 and 82.500 , respectively. According to the chi-square test, the difference between the two groups was significant $(P \leq 0.05)$. The mean of systolic blood pressure in patients with severe and moderate pain was 122.321 and 117.500 , respectively. This difference was statistically significant $(P=0.038)$. The mean of diastolic blood pressure in patients with moderate and severe pain was 76.47 and 71.632 , respectively. This difference was statistically significant $(P=0.023)$. The respiratory rate of patients with severe and moderate pain was 17.29 and 15.99, respectively, and there was a significant difference between the two groups in terms of the respiratory rate $(P \leq 0.05)$ (Table 1$)$.

The mean of pain reduction was 0.471 in the group with severe pain after 15 minutes after drug administration. This value was 0.878 in the group with moderate pain. The $t$ test analysis showed a significant difference in pain relief between the two groups $(P \leq 0.05)$. After 30 minutes, the intensity of pain reduction in patients with severe and moderate pain was 1.124 and 1.796 , respectively. This difference in pain relief was still significant $(P \leq 0.05)$. However, the reduction in pain in patients with moderate pain, according to the protocol, was more than the minimum acceptable level of $13 \mathrm{~mm}$ according to the 
Table 1. Comparison of baseline characteristics between the two groups (patients with severe and moderate pain)

\begin{tabular}{|c|c|c|c|}
\hline Variables & Severe pain $(7-10)$ & Moderate pain (4-6) & $P$ value \\
\hline Male, \% & 64.4 & 65.3 & \multirow{2}{*}{0.95} \\
\hline Female, \% & 35.6 & 35.7 & \\
\hline Mean age (SD) & $37.18 \pm 11.68$ & $37.836 \pm 12.45$ & 0.76 \\
\hline Accidents related to Transportation, $\%$ & 74.1 & 73.5 & \multirow{2}{*}{0.93} \\
\hline Accidents not related to transportation, $\%$ & 25.9 & 26.5 & \\
\hline Systolic blood pressures (mm Hg) & 124.321 & 117.500 & 0.038 \\
\hline Diastolic blood pressures $(\mathrm{mm} \mathrm{Hg})$ & 76.470 & 71.632 & 0.023 \\
\hline Pulse rate $(1 / \mathrm{min})$ & 90.172 & 82.688 & 0.001 \\
\hline Respiratory rate (1/min) & 17.305 & 15.081 & 0.001 \\
\hline
\end{tabular}

VAS scale. Pain reduction 45 minutes after the injection of ketorolac ampule in severely injured patients was 2.636 . This rate of pain reduction according to the pain reduction protocol was higher than the maximum acceptable level of $13 \% \mathrm{~mm}$ according to the VAS scale. In patients with a mean pain of 3.122 , this reduction was greater than $30 \mathrm{~mm}$ and it was medically acceptable. However, the difference in pain relief between the two groups of patients with severe and moderate pain was statistically significant $(P \leq 0.05)$ (Table 2).

\section{Discussion}

Based on the results obtained from the current study ketorolac can be used to control pain in trauma patients who have moderate pain after 10 to 15 minutes after the onset of the pain both in the short term and in the long term. Ketorolac is not a good drug for controlling the pain of severely injured patients due to the onset of peak effects more than 30 minutes. Conversely, ketorolac can be prescribed in periods of less than 30 minutes due to minor side effects (Figure 1). Similar results were reported in the study conducted by Ellerton et al, in which they recommended that ketorolac can be used to control the pain in mountain accidents due to the onset of action after more than 30 minutes. The results of this study are consistent with the results of the present study (10). Delavar Kasmaei et al revealed that ketorolac and magnesium sulfate can be both effective in controlling migraine pain, but ketorolac

Table 2. Comparing the study outcomes and pain severity at different times for the two groups

\begin{tabular}{|c|c|c|c|c|c|c|}
\hline \multirow{2}{*}{ Variables } & \multirow{2}{*}{ Time } & \multicolumn{4}{|c|}{ Study groups } & \multirow{2}{*}{$P$ value } \\
\hline & & \multicolumn{2}{|c|}{ Severe pain } & \multicolumn{2}{|c|}{ Moderate pain } & \\
\hline \multirow{4}{*}{ Pain severity } & $0 \mathrm{~min}$ & 8.376 & (Pain relief) & 5.122 & (Pain relief) & - \\
\hline & $15 \mathrm{~min}$ & 7.905 & 0.471 & 4.244 & 0.878 & 0.001 \\
\hline & $30 \mathrm{~min}$ & 7.252 & 1.124 & 3.326 & 1.796 & 0.001 \\
\hline & $45 \mathrm{~min}$ & 5.740 & 2.363 & 2.000 & 3.122 & 0.001 \\
\hline \multirow{4}{*}{ Systolic BP $(\mathrm{mm} \mathrm{Hg}) \pm S \mathrm{SD}$} & $0 \mathrm{~min}$ & \multicolumn{2}{|c|}{$124.321 \pm 19.85$} & \multicolumn{2}{|c|}{$17.29 \pm 117.500$} & 0.038 \\
\hline & $15 \mathrm{~min}$ & \multicolumn{2}{|c|}{$125.308 \pm 18.93$} & \multicolumn{2}{|c|}{$17.35 \pm 117.945$} & 0.026 \\
\hline & $30 \mathrm{~min}$ & \multicolumn{2}{|c|}{$125.925 \pm 16.78$} & \multicolumn{2}{|c|}{$16.25 \pm 119.543$} & 0.034 \\
\hline & $45 \mathrm{~min}$ & \multicolumn{2}{|c|}{$125.432 \pm 15.65$} & \multicolumn{2}{|c|}{$14.84 \pm 120.281$} & 0.10 \\
\hline \multirow{4}{*}{$\begin{array}{l}\text { Diastolic BP }(\mathrm{mm} \\
\mathrm{Hg}) \pm \mathrm{SD}\end{array}$} & $0 \mathrm{~min}$ & \multicolumn{2}{|c|}{$76.740 \pm 11.51$} & \multicolumn{2}{|c|}{$71.632 \pm 12.13$} & 0.023 \\
\hline & $15 \mathrm{~min}$ & \multicolumn{2}{|c|}{$76.790 \pm 11.02$} & \multicolumn{2}{|c|}{$72.653 \pm 11.86$} & 0.037 \\
\hline & $30 \mathrm{~min}$ & \multicolumn{2}{|c|}{$78.352 \pm 10.21$} & \multicolumn{2}{|c|}{$73.877 \pm 11.14$} & 0.023 \\
\hline & $45 \mathrm{~min}$ & \multicolumn{2}{|c|}{$75.409 \pm 10.99$} & \multicolumn{2}{|c|}{$75.409 \pm 10.99$} & 0.11 \\
\hline \multirow{4}{*}{ Pulse rate $(1 / \mathrm{min}) \pm \mathrm{SD}$} & $0 \mathrm{~min}$ & \multicolumn{2}{|c|}{$90.047 \pm 11.19$} & \multicolumn{2}{|c|}{$82.693 \pm 8.22$} & 0.001 \\
\hline & $15 \mathrm{~min}$ & \multicolumn{2}{|c|}{$89.917 \pm 10.24$} & \multicolumn{2}{|c|}{$82.367 \pm 7.71$} & 0.001 \\
\hline & $30 \mathrm{~min}$ & \multicolumn{2}{|c|}{$88.364 \pm 9.09$} & \multicolumn{2}{|c|}{$80.335 \pm 6.97$} & 0.001 \\
\hline & $45 \mathrm{~min}$ & \multicolumn{2}{|c|}{$85.963 \pm 8.88$} & \multicolumn{2}{|c|}{$78.511 \pm 6.04$} & 0.001 \\
\hline & $0 \mathrm{~min}$ & \multicolumn{2}{|c|}{$17.305 \pm 2.75$} & & & 0.001 \\
\hline Respiratory rate (1/ & $15 \mathrm{~min}$ & & & & & 0.001 \\
\hline $\min ) \pm S D$ & $30 \mathrm{~min}$ & & & & & 0.001 \\
\hline & $45 \mathrm{~min}$ & & & & & 0.001 \\
\hline
\end{tabular}




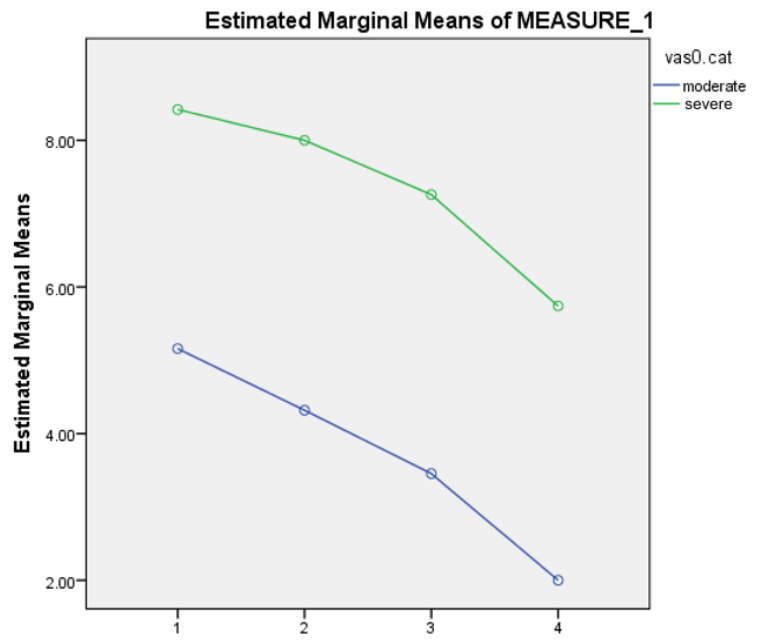

Figure 1. Mean of pain severity at various times in the two groups of patients with severe and moderate pain.

was less effective than magnesium sulfate (11). Findings of a systematic review by Yousefifard et al showed that of the 12 guidelines developed for the management of severe pain $(\mathrm{VAS}=10-7)$ in the pre-hospital setting for adults, only two guidelines recommended the use of ketorolac for severe pain. Also out of 10 comprehensive guidelines for moderate pain management $(\mathrm{VAS}=4-6)$, only one guideline recommended controlling pain with ketorolac. The final results of this study showed that the first line of medication for severe and moderate pain management in a hospital setting for adults and children is fentanyl and morphine (9). Neri et al compared ketorolac sublingual with tramadol ampoule in children with severe pain due to mandibular fracture and found that ketorolac and tramadol both can be effective for pain control. This finding is inconsistent with our results which can be due to differences in the research environment and the age difference between the individuals (12). Mallinson considered factors such as the slow onset of ketorolac and concluded that ketorolac could be a suitable drug in pre-hospital environments where the transfer time of the patient is less than 1 hour (7). In contrast, Mahshidfar et al in a study conducted in a prehospital setting in Tehran province compared the effectiveness of ketorolac and acetaminophen injections and concluded that both drugs could control pain in patients (13). However, in a doubleblind clinical trial to compare the efficacy of morphine sulfate and ketofol, the target population was patients suffering from severe renal pain resistant to $30 \mathrm{mg}$ injection of ketorolac (14). Eftekharian and Ilkhani Pak selected 50 patients who underwent surgery for mandibular jaw fractures. They evaluated the effectiveness of ketorolac in the two groups of ketorolac $(n=25)$ and placebo $(n=25)$. They concluded that ketorolac is an effective drug for pain management after surgery with minimal transient side effects (15).

\section{Limitations}

Due to lack of effective painkillers such as opioids in the pre-hospital setting of Iran at present, in this study it was not feasible to conduct a randomized double-blind study. Also, after the delivery of the injured patients by 115 emergency personnel to the hospital, patients left the prehospital emergency system. Thus, it was not possible to continue monitoring and evaluating the injured patients in terms of pain intensity and drug side effects.

\section{Conclusion}

Based on the results of the present study, it can be concluded that ketorolac is suitable for controlling moderate pain and severe pain in trauma patients due to the onset of the effects after 10 to 15 minutes. On the other hand, due to the peak effect (more than 30 minutes), it is not an effective drug for trauma patients with severe pain in a short period. However due to the distance and duration of the transfer of trauma patients from road emergency bases, a strong drug or multimodal drug can be used to reduce severe pain.

\section{Authors' contribution}

SMA (Correspondent author) has conducted the research and write the initial manuscript. MY supervised the study. SZ and MN participated in statistical analysis. SMA and $\mathrm{ZM}$ have revised the manuscript.

The final version of the manuscript was approved by all authors.

\section{Ethical issues}

In this study, we recruited the EMS staff working in the prehospital emergency department of Lorestan province, Iran. Participants completed the consent form before the start of the study. The study protocol was approved by the ethics committee of Shahid Beheshti University of Medical Sciences, Iran with code number IR.SBMU.PHARMACY. REC.1397.265.

\section{Acknowledgements}

The authors would like to appreciate all the pre-hospital emergency staff working in pre-hospital emergency stations of Lorestan province.

\section{References}

1. Rhee P, Joseph B, Pandit V, Aziz H, Vercruysse G, Kulvatunyou $\mathrm{N}$, et al. Increasing trauma deaths in the United States. Ann Surg 2014; 260(1): 13-21. doi: 10.1097/ sla.0000000000000600.

2. Ahmadi A, Bazargan-Hejazi S, Heidari Zadie Z, Euasobhon P, Ketumarn P, Karbasfrushan A, et al. Pain management in trauma: a review study. J Inj Violence Res 2016; 8(2): 89-98. doi: 10.5249/jivr.v8i2.707.

3. Dijkstra BM, Berben SA, van Dongen RT, Schoonhoven L. Review on pharmacological pain management in trauma patients in (pre-hospital) emergency medicine 
in the Netherlands. Eur J Pain 2014; 18(1): 3-19. doi: 10.1002/j.1532-2149.2013.00337.x.

4. Khan MS, Shuaib W, Evans DD, Swain FR, Alweis R, Mehta AS, et al. Evidence-based practice: best imaging practice in musculoskeletal disorders. J Trauma Nurs 2014; 21(4): 1709. doi: 10.1097/jtn.0000000000000059.

5. Berben SA, Meijs TH, van Grunsven PM, Schoonhoven L, van Achterberg T. Facilitators and barriers in pain management for trauma patients in the chain of emergency care. Injury 2012; 43(9): 1397-402. doi: 10.1016/j. injury.2011.01.029.

6. Katzung BG, Trevor AJ. Basic \& Clinical Pharmacology, SMARTBOOK ${ }^{\mathrm{T}}$. McGraw Hill Professional; 2014.

7. Mallinson DTE. A review of ketorolac as a prehospital analgesic. Journal of Paramedic Practice 2019; 11(11): 1-6. doi: 10.12968/jpar.2019.11.11.CPD1.

8. Farahmand S, Shafazand S, Alinia E, Bagheri-Hariri S, Baratloo A. Pain management using acupuncture method in migraine headache patients; a single blinded randomized clinical trial. Anesth Pain Med 2018; 8(6): e81688. doi: 10.5812/aapm.81688.

9. Yousefifard M, Askarian-Amiri S, Madani Neishaboori A, Sadeghi M, Saberian P, Baratloo A. Pre-hospital pain management; a systematic review of proposed guidelines. Arch Acad Emerg Med 2019; 7(1): e55.

10. Ellerton J, Milani M, Blancher M, Zen-Ruffinen G, Skaiaa
SC, Brink B, et al. Managing moderate and severe pain in mountain rescue. High Alt Med Biol 2014; 15(1): 8-14. doi: 10.1089/ham.2013.1135.

11. Delavar Kasmaei H, Amiri M, Negida A, Hajimollarabi S, Mahdavi N. Ketorolac versus magnesium sulfate in migraine headache pain management; a preliminary study. Emerg (Tehran) 2017; 5(1): e2.

12. Neri E, Maestro A, Minen F, Montico M, Ronfani L, Zanon $\mathrm{D}$, et al. Sublingual ketorolac versus sublingual tramadol for moderate to severe post-traumatic bone pain in children: a double-blind, randomised, controlled trial. Arch Dis Child 2013; 98(9): 721-4. doi: 10.1136/archdischild-2012-303527.

13. Mahshidfar B, Rezai M, Abbasi S, Farsi D, Hafezimoghadam $\mathrm{P}$, Mofidi M, et al. Intravenous acetaminophen vs. ketorolac in terms of pain management in prehospital emergency services: a randomized clinical trial. Adv J Emerg Med 2019; 3(4): e37. doi: 10.22114/ajem.v0i0.130.

14. Faridaalaee G, Mohammadi N, Merghati SZ, Keyghobadi Khajeh F, Naghipour B, Pouraghaei M, et al. Intravenous morphine vs intravenous ketofol for treating renal colic; a randomized controlled trial. Emerg (Tehran) 2016; 4(4): 202-6.

15. Eftekharian HR, Ilkhani Pak H. Effect of intravenous ketorolac on postoperative pain in mandibular fracture surgery; a randomized, double-blind, placebo-controlled trial. Bull Emerg Trauma 2017; 5(1): 13-7. 